\title{
Constructing a Probabilistic Model for Automated Liver Region Segmentation Using Non-contrast X-Ray Torso CT images
}

\author{
Xiangrong Zhou ${ }^{1}$, Teruhiko Kitagawa ${ }^{1}$, Takeshi Hara ${ }^{1}$, Hiroshi Fujita ${ }^{1}$, \\ Xuejun Zhang ${ }^{1}$, Ryujiro Yokoyama ${ }^{2}$, Hiroshi Kondo ${ }^{2}$, Masayuki Kanematsu ${ }^{2}$, \\ and Hiroaki Hoshi ${ }^{2}$ \\ ${ }^{1}$ Department of Intelligent Image Information, Division of Regeneration and Advanced \\ Medical Sciences, Graduate School of Medicine, Gifu University, Gifu 501-1194, Japan \\ \{zxr, kitagawa, hara, fujita, zhang\}@fjt.info.gifu-u.ac.jp \\ ${ }^{2}$ Department of Radiology, Gifu University School of Medicine and University Hospital, \\ Gifu 501-1194, Japan
}

\begin{abstract}
A probabilistic model was proposed in this research for fullyautomated segmentation of liver region in non-contrast X-ray torso CT images. This probabilistic model was composed of two kinds of probability that show the location and density (CT number) of the liver in CT images. The probability of the liver on the spatial location was constructed from a number of CT scans in which the liver regions were pre-segmented manually as gold standards. The probability of the liver on density was estimated specifically using a Gaussian function. The proposed probabilistic model was used for automated liver segmentation from non-contrast CT images. 132 cases of the CT scans were used for the probabilistic model construction and then this model was applied to segment liver region based on a leave-one-out method. The performances of the probabilistic model were evaluated by comparing the segmented liver with the gold standard in each CT case. The validity and usefulness of the proposed model were proved.
\end{abstract}

\section{Introduction}

With the development of the CT technology, the whole body scans using X-ray CT or MRI scanner are practicable for the clinical purpose. Now, the radiologists can easily make a volumetric torso X-ray CT scan in as little as 20-30 seconds to examine health-related issues such as vascular problems, liver problems and the detection of lesions in different organs and tissue within the torso region. However, the interpretation for such a torso CT scan that includes over 1000 axial slices of CT images on a screen or films without any oversight is tedious for radiologists, The computer-aided diagnosis (CAD) system that can show the 3-D anatomical structure of human body and find out the location of the suspicious regions is strongly expected to reduce the burden and increase the accuracy of the medical image interpretation.

Among the different organ and tissue regions, liver is one of the most important diagnosis target organs of the CAD system. Lesion detection and surgery planning of liver always require the CAD system to extract the liver region firstly from CT images. However, without any prior knowledge or assistance of human operators, the 
traditional image processing methods (such as gray-level thresholding, region growing, etc.) can not provide a reliable automated extraction of liver region especially in non-contrast CT images. The prior knowledge (always appears as an atlas) that shows the spatial location, shape and density (CT number) of liver region are required as the assistance for the automated liver segmentation process.

In order to provide the prior knowledge for abdominal organ segmentations, Park et al. proposed a method for abdominal probabilistic atlas construction [1] using the contrast abdominal CT images and some further approaches were also reported $[2,3]$. However, how to use the atlas to realize a fully-automated liver segmentation could not be solved completely and the performance evaluations of such atlases were limited based on a small database [1,2]. In this paper, we proposed a method to estimate the liver probability in each of CT cases specifically and use the estimated probability to segment the liver region automatically. The performance of the estimated liver probability and liver segmentation results were evaluated by a coincidence ratio measurement and a characteristic curve analysis based on manual extraction results of liver regions in 132 normal liver CT cases and 20 abnormal CT cases. In the following sections, we firstly describe the outline of our liver segmentation method in Section 2, and then, show the details of liver probability estimation method in Section 3. Experimental results are presented in Section 4 and discussions on the accuracy evaluations of the probability estimation and liver segmentation are described in Section 5. Finally, a conclusion is given in Section 6.

\section{Outline of the Liver Segmentation}

The outline of our proposed liver region segmentation scheme is shown in Fig. 1. The input of the scheme is a non-contrast CT scan covering human torso region with an isotopic spatial resolution. The output of the scheme is a 3-D binary image in which the voxels of the liver region are labeled with ' 1 ' and the voxels of other regions are labeled with ' 0 '. The proposed scheme is composed of 2 principal steps: (1) Automated estimation of the liver probability in the inputted CT images. After this step, each voxel in the CT images is attached with a value (0.0-1.0) showing the probability belongs to the liver region. (2) Automated segmentation of liver region in CT images based on the estimated liver probability.

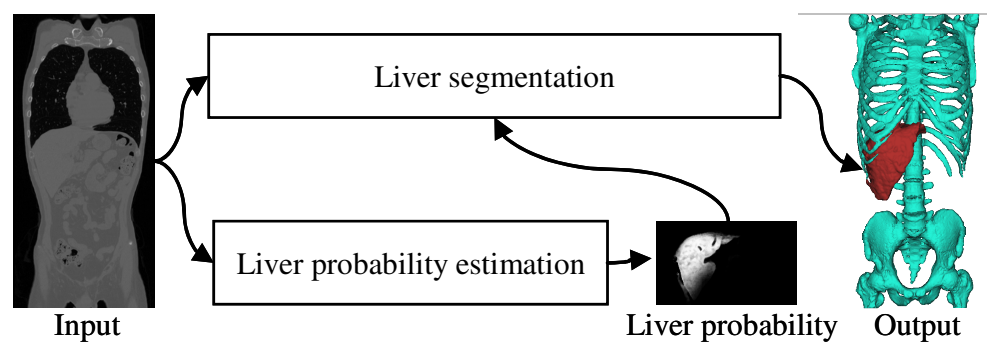

Fig. 1. Outline of our automated liver segmentation scheme 


\section{Automated Estimation of Liver Probability}

\subsection{Definition of the Probabilistic Liver Model}

The simplest model of liver region (described as event $A$ ) can be considered as a 3-D connected component that appears in a special location (event $B$ ) of human body with a special CT number (event $C$ ) in CT images. We define the $P(A)$ as the probability of each voxel that belongs to liver region on CT images, $P(B)$ as the probability of liver location in the spatial anatomical structure of human torso, and $P(C \mid B)$ as the probability of the density (CT number) in liver region on CT images. Using the above definitions, we can get the following equation.

$$
P(A)=P(B \cap C)=P(B) \times P(C \mid B)
$$

The liver probability $P(A)$ for a special CT scan can be calculated by $P(B)$ [probability of the anatomical location of liver region in human torso] and $P(C \mid B)$ [probability of liver density on CT images]. This research tries to develop the methods to estimate the $P(B)$ and $P(C \mid B)$ dynamically and generate the probability $P(A)$ for liver segmentation.

\subsection{Construction of a Liver Atlas}

The liver atlas that used in this research is defined as a probability of liver location (includes shape and position) under a normalized anatomical structure of human body [3]. In order to guarantee the correctness and accuracy of the atlas, arranging the anatomical structure (composed of bone frame, diaphragm, and body surface) in different CT cases and investigating the variation of the liver locations based on a large number of the CT cases are necessary. A standard anatomical structure surrounding the liver region is defined for liver region arrangements [3]. We deform the anatomical structures in different CT cases to the position of the standard anatomical structure firstly, and then vote the liver region in each CT case to the spatial positions of the standard anatomical structure. The voting result that shows the liver probabilities in each spatial position in the standard anatomical structure is regarded as the liver atlas [3].

\subsection{Estimation of the Liver Probability on Spatial Location}

Using the constructed liver atlas in Section 3.2, we developed a procedure to estimate the probability of the liver region in an inputted CT case automatically as shown in Fig.2. This procedure included following 3 processing steps: (1): The bone region was extracted using a gray-level thresholding method [5] and the diaphragm was identified based on the shape of air regions inside of lung [6]. (2): Using a calibration of the landmarks (composed of 8 vertexes of a circumscribed hexahedron of bone frame and body surface, 200-300 sampling points of diaphragm surface) between the inputted CT cases and standard anatomical structure [3], a transformation matrix of the Thinplate spline (TPS) [4] was calculated. (3): The liver atlas in the standard anatomical structure was deformed by TPS using the calculated transformation matrix to show the probability $P(B)$ of liver location in the inputted CT images [Fig.2]. 


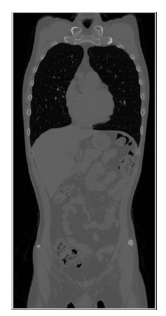

CT image

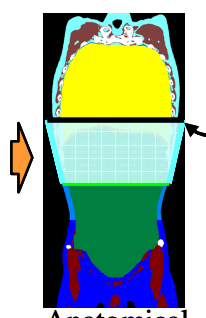

Anatomical structure

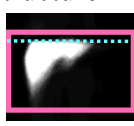

Liver atlas
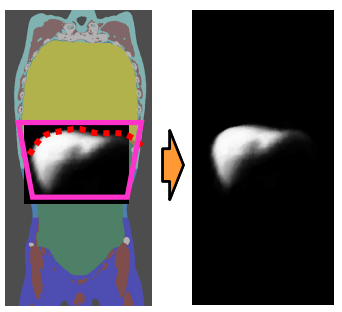

Probability of liver location

Fig. 2. Estimation of the liver probability on spatial location $(P(B))$ based on liver atlas

\subsection{Estimation of the Liver Probability on Density}

After the $P(B)$ estimation, we proposed a method to estimate the $P(C \mid B)$ that can be considered as the liver probability on density (CT number) feature. We assumed a Gaussian distribution to approximate the density distribution of liver by the following equation.

$$
p\left(y_{i} \mid i \in \text { Liver }\right)=\frac{1}{\sqrt{2 \pi \sigma^{2}}} \exp \left(\frac{-\left(y_{i}-\mu\right)^{2}}{2 \sigma^{2}}\right)
$$

The Gaussian parameters could be estimated by observing the density histogram of the region that each voxel $i$ should satisfy the condition $\left[P_{i}(B)>0\right]$. The $\mathrm{CT}$ number which was the maximum peak in the histogram was selected as the mean value $\mu$ and a Gaussian distribution $N(\mu, \sigma)$ which has the same FWHM (full width half maximum) with the observed density histogram was decided. Then, we calculated the

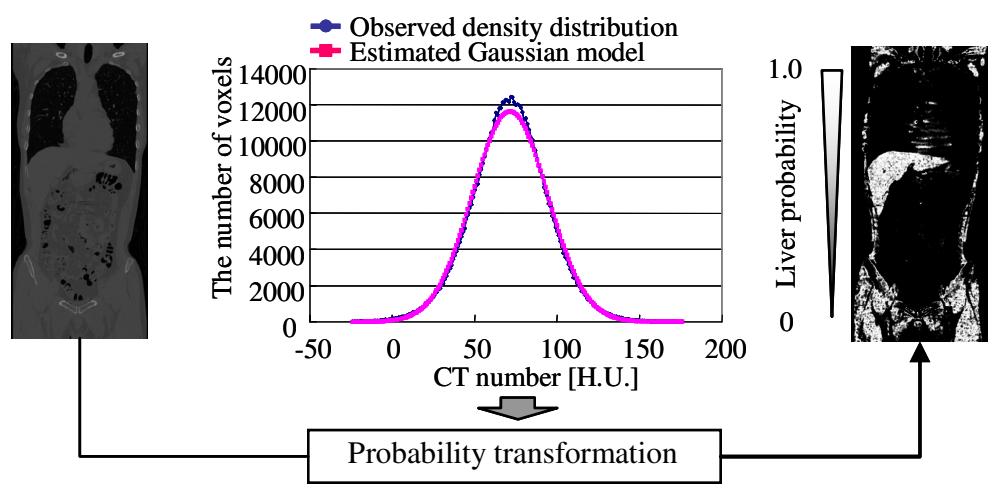

Fig. 3. Estimation of the liver probability on density $(P(C \mid B))$ 
$P(C \mid B)$ from the $\mathrm{CT}$ images by a probability transformation using the $N(\mu, \sigma)$ as a characteristic curve [Fig.3]. A likelihood image was generated to show the probability $P(C \mid B)$ which indicates the probability of liver on CT numbers [Fig.3].

\subsection{Calculating the Liver Probability for Automated Segmentation}

The probability $P(A)$ for CT images can be obtained simply by a multiplication of $P(B)$ and $P(C \mid B)$. In fact, we generated a total likelihood image to show the liver probability $P(A)$ by an image multiplication. Based on the $P(A)$, the liver region can be extracted by simply selecting the voxel $i$ that satisfied the condition $\left[P_{i}(A)>t h\right]$, and then, the binary regions were refined by a binary morphological processing [using a ball kernel with a radius $=r$ ]. At last, the biggest connected component in 3-D was decided as the liver region. The procedure was designed in a fully-automatic mode without any assistance by operators.

\section{Experiments}

132 normal liver cases of non-contrast torso CT images were used in experiment. Each CT case has an isotopic spatial resolution of about $0.6 \mathrm{~mm}$ and density (CT number) resolution of 12 bits. The ground truth of liver region (gold standard) in each CT case was instructed by two experienced radiologists (authors H.K. and M.K.) using a semi-automatic segmentation method for atlas construction and accuracy evaluation for segmentation results. The performance of our method was evaluated based on a leave-one-out method that selects 131 cases to construct a liver atlas and use it to segment the liver region from the surplus 1 case. The proposed liver segmentation method was also applied to the additional 20 abnormal liver cases (fat liver) using the constructed liver atlas from 132 normal cases. The parameters $(t h=0.03, r$ $=2$ (voxels)) were determined by our experience in liver segmentation. The experimental results of one CT case is shown in Fig.4.

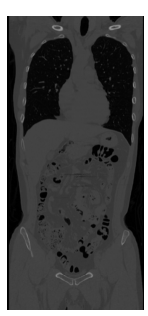

(a)

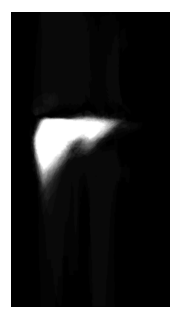

(b)

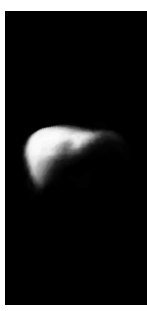

(c)

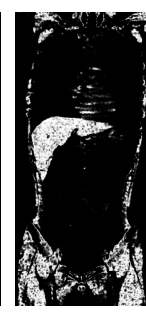

(d)

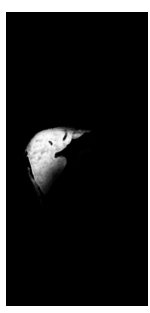

(e)

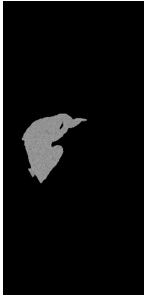

(f)

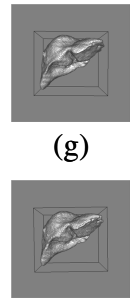

(h)

Fig. 4. An example of liver segmentation results. (a) original CT image (1 coronal slice), (b) liver atlas, (c) $P(B)$ : liver probability on spatial location, (d) $P(C \mid B)$ : liver probability on density, (e) $P(A)$ : liver probability, (f) liver segmentation result (1 coronal slice), (g) ground truth of liver (3-D view), (h) liver segmentation result (3-D view). 
The coincidence ratio between the segmented liver region and the ground truth of liver region was used to evaluate the accuracy of the liver segmentation method. A characteristic curve analysis shows the relationship between the liver extraction ratio and over extraction ratio of liver was used to evaluate the performance and accuracy of proposed liver model (liver probabilities).

\section{Discussion}

We found that the liver region was segmented correctly in each of CT cases. The mean value of the coincidence ratio between segmentation results and gold standards was 0.932 for normal liver cases and 0.898 for fatty liver cases, respectively. The results showed that the proposed method has the ability to segment the liver region in non-contrast CT images even for the abnormal liver cases. The error was caused by the misrecognition of a part of muscles and other tissues around the liver regions. Refining the liver segmentation results based on edge information of liver will be applied to reduce such error in the future.

The performance and accuracy of the estimated liver probability were evaluated by the described characteristic curve analysis which is similar to the ROC analysis [Fig.5]. The area $A$ under the curve was used as the evaluation standard. The mean value of the $A$ was 0.99 with the standard deviation of 0.02 for 132 normal cases using leave-one-out method [Fig.5]. We confirmed that the liver probability estimation based on liver atlas can provide satisfied information for liver segmentation from noncontrast CT images.

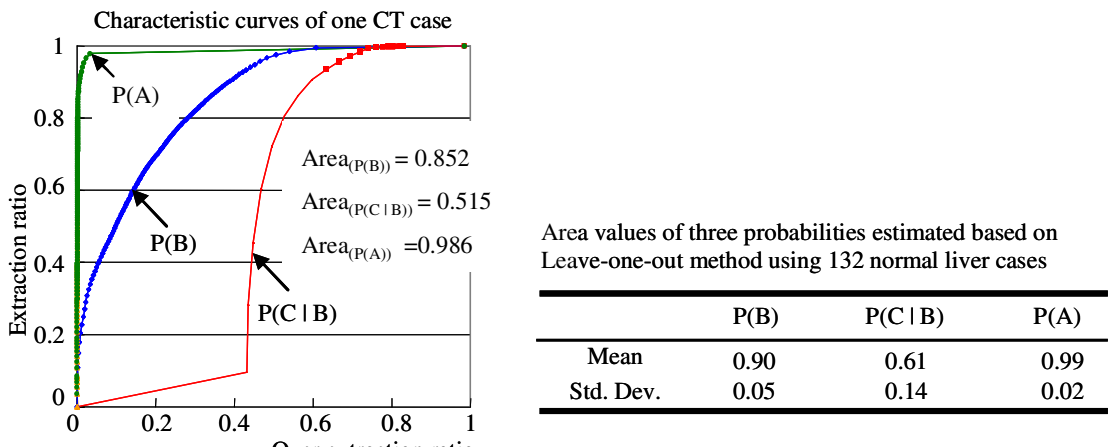

Fig. 5. Performance evaluation results of the liver probabilities using a characteristic curve analysis

We found the density distribution of the ground truth of liver region in each CT case was quite close to a Gaussian distribution [Fig.2]. However, the mean value and standard deviation of the liver density in each case varied largely. Using our Gaussian parameter estimation, we confirmed the mean value of the coincidence ratios between the real density distribution and estimated Gaussian model was about $94 \%$. The 
margin of the error was caused by the vessels that were recognized incorrectly as a part of liver region during the semi-automatic segment process. The experiential result showed that the density of liver region can be presented by a Gaussian distribution and Gaussian parameters should be estimated respectively from the CT images for the different patient cases.

Normalizing the liver location in different patient cases was very important before the liver atlas construction; we compared the results of the liver atlas with and without the normalization [Fig.6]. We measured the convergence of probability of liver location and confirmed that our method (warping the diaphragm and bone structure to reduce the variance of liver location and shape in different CT images) was very effective to improve the accuracy of the atlas on liver location [Fig.6].

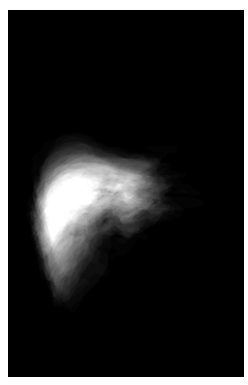

(a)

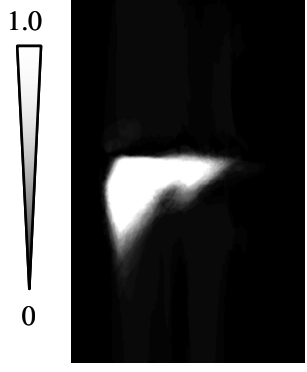

(b)

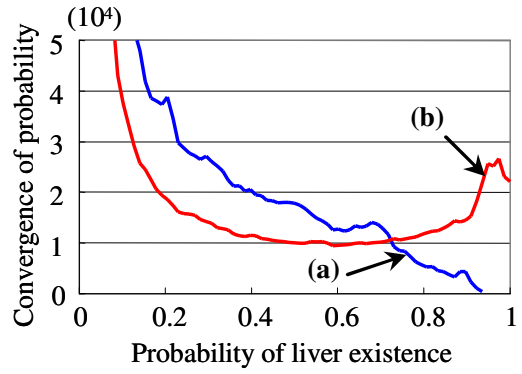

(c)

Fig. 6. Liver atlas construction results (a) liver atlas without anatomical structure arrangements, (b) liver atlas with anatomical structure arrangements, (c) probability convergences of the (a) and (b)

\section{Conclusion}

We proposed a method to construct a probabilistic liver model for automated liver segmentation from non-contrast CT images. The proposed method was applied to segment liver regions in $152 \mathrm{CT}$ cases for performance evaluations. We confirmed that the proposed liver model can improve the robustness and effectiveness of liver segmentation process and provide a feasible solution to extract the liver region from the non-contrast CT images automatically.

\section{Acknowledgements}

Authors thank to members of Fujita Laboratory and especially to Dr. G. Lee for her valuable discussion. This research was supported in part by research grants of Grantin-Aid for Scientific Research on Priority Areas, in part by Ministry of Health, Labour, and Welfare under a Grant-In-Aid for Cancer Research, and in part by the Knowledge Cluster Initiative of the MEXT, Japanese Government. 


\section{References}

[1] H.Park, P.H. Bland, and C.R. Meyer: Construction of an Abdominal Probabilistic Atlas and its Application in Segmentation, IEEE Trans. Med. Imag. 22 (4) (2003) 483-492.

[2] H.Kobatake, A. Shimizu, X. Hu, et al.: Simultaneous Segmentation of Multiple Organs in Multi-Dimensional Medical Images, Proc. of the First International Symposium on Intelligent Assistance in Diagnosis of Multi-dimensional Medical Images, Part I, (2005) 11-18.

[3] X.Zhou, T.Kitagawa, K.Okuo, et al.: Construction of a Probabilistic Atlas for Automated Liver Segmentation in Non-contrast Torso CT Images, Proc. of the 19th International Congress and Exhibition CARS 2005, International Conference Series 1281, Elsevier (2005), 1163-1168.

[4] F. L. Bookstein: Principal Warps:Thin-Plate Splines and the Decomposition of Deformations, IEEE Trans. PAMI, 11 (6) (1989) 567-585.

[5] X. Zhou, T. Hara, H. Fujita, et al.: Automated Segmentations of Skin, Soft-tissue and Skeleton from Torso CT images, Proc. of SPIE-Medical Imaging 2004, vol. 5370, (2004) 1634-1639.

[6] X. Zhou, T. Hara, H. Fujita, et al.: Preliminary study for automated recognition of anatomical structure from torso CT images, Proc. of the 2005 IEEE Engineering in Medicine and Biology 27th Annual Conference, Shanghai, China, paper\#340, (2005). 